Acta Crystallographica Section D

Biological

Crystallography

ISSN 0907-4449

Arnau Casañas, ${ }^{a} \ddagger \S$ Jordi QuerolAudí, ${ }^{a} \neq$ Pablo Guerra, ${ }^{a}$ Joan Pous, ${ }^{\text {b,c }}$ Hideaki Tanaka, Tomitake Tsukihara, ${ }^{\mathrm{d}}$ Nuria Verdaguer $^{\mathrm{a} *}$ and Ignasi Fita ${ }^{\mathrm{a} *}$

anstitut de Biologia Molecular de Barcelona (CSIC), Parc Científic de Barcelona, Baldiri i

Reixac 10, 08028 Barcelona, Spain,

b Plataforma Automatizada de Cristalografía,

Parc Científic de Barcelona, Baldiri i Reixac 10, 08028 Barcelona, Spain, ' IRB Barcelona, Parc Científic de Barcelona, Baldiri i Reixac 10, 08028 Barcelona, Spain, and Institute for Protein Research, Osaka University,

3-2 Yamadaoka, Suita, Osaka 565-0871, Japan

‡ Joint first authors.

$\S$ Current address: Institute of Molecular Biology and Biophysics, ETH Zurich, Schafmattstrasse 20, HPK H10, 8093 Zurich, Switzerland.

Correspondence e-mail: nvmcri@ibmb.csic.es, ifrcri@ibmb.csic.es

\title{
New features of vault architecture and dynamics revealed by novel refinement using the deformable elastic network approach
}

The vault particle, with a molecular weight of about $10 \mathrm{MDa}$, is the largest ribonucleoprotein that has been described. The $\mathrm{X}$-ray structure of intact rat vault has been solved at a resolution of $3.5 \AA$ [Tanaka et al. (2009), Science, 323, 384388], showing an overall barrel-shaped architecture organized into two identical moieties, each consisting of 39 copies of the major vault protein (MVP). The model deposited in the PDB includes 39 MVP copies (half a vault) in the crystal asymmetric unit. A $2.1 \AA$ resolution structure of the seven $\mathrm{N}$-terminal repeats (R1-7) of MVP has also been determined [Querol-Audí et al. (2009), EMBO J. 28, 3450-3457], revealing important discrepancies with respect to the MVP models for repeats $\mathrm{R} 1$ and $\mathrm{R} 2$. Here, the re-refinement of the vault structure by incorporating the high-resolution information available for the R1-7 domains, using the deformable elastic network (DEN) approach and maintaining strict 39-fold noncrystallographic symmetry is reported. The new refinement indicates that at the resolution presently available the MVP shell can be described well as only one independent subunit organized with perfect $D 39$ molecular symmetry. This refinement reveals that significant rearrangements occur in the $\mathrm{N}$-terminus of MVP during the closing of the two vault halves and that the 39 -fold symmetry breaks in the cap region. These results reflect the highly dynamic nature of the vault structure and represent a necessary step towards a better understanding of the biology and regulation of this particle.

\section{Introduction}

The vault complex, with overall dimensions of $40 \times 40 \times$ $70 \mathrm{~nm}$ and a mass of $10 \mathrm{MDa}$ (Tanaka \& Tsukihara, 2012), is the largest ribonucleoprotein particle that has been found in eukaryotes (Kedersha et al., 1990; Hamill \& Suprenant, 1997; Herrmann et al., 1997). The main component of vaults is the $100 \mathrm{kDa}$ major vault protein (MVP; Kedersha \& Rome, 1986), whereas the two minor vault proteins comprise the $193 \mathrm{kDa}$ vault poly(ADP-ribose) polymerase (VPARP; Kickhoefer, Siva et al., 1999) and the $240 \mathrm{kDa}$ telomerase-associated protein-1 (TEP1; Kickhoefer, Stephen et al., 1999). Small untranslated RNAs (vRNAs), the number of which varies among species, are also found as constitutive components (Kickhoefer et al., 1993; Stadler et al., 2009). Vaults have been associated with intrinsic drug resistance (Scheffer et al., 1995), but have also been related to nucleocytoplasmatic transport, cell signalling and innate immunity (Berger et al., 2009; Kowalski et al., 2007). As MVP knockdown results in disruption of the lysosomal compartment, it has been proposed that vault complexes might participate in drug resistance by their influence on the lysosomal function (Herlevsen et al., 2007). Interestingly, vaults disassemble into halves at low $\mathrm{pH}$
Received 27 November 2012 Accepted 14 February 2013

PDB Reference: Re-refinement of the vault ribonucleoprotein particle, $4 \mathrm{hl} 8$ 
(Goldsmith et al., 2007; Esfandiary et al., 2009). The possibility of the acidic nature of lysosomes participating in the opening of vault particles has still to be explored.

Their hollow structure, rapid movements and distinct subcellular localization led to the hypothesis that vaults might represent rather promiscuous transport vehicles (Steiner et al., 2006). Recent experiments have shown that recombinant vaults are also capable of half-vault exchange mechanisms in vitro (Yang et al., 2010). These experiments indicate that vaults are not rigid impenetrable boxes, but a fluctuating dynamic structure presenting substantial flexibility. Furthermore, the proposal that vaults could act as a cellular transporter implies that these particles must present a highly regulated opening mechanism in order to efficiently incorporate and deliver the specific vault cargo. Even so, both the precise regulation of the opening mechanism and the structural characteristics that can allow such surprising dynamics still have to be clearly defined.

The X-ray structure of rat vault was solved to a resolution of $3.5 \AA$ (Tanaka et al., 2009). It shows that the vault shell is organized into two identical moieties, each consisting of 39 copies of MVP (Fig. 1a). Within the vault shell, MVP is organized into 12 domains: nine structural repeat domains at the N-terminus, followed by a shoulder $\alpha / \beta$ domain, a caphelix and a cap-ring at the C-terminus (Fig. 1b). In this structure the initial phasing was achieved by density modification, in particular using noncrystallographic symmetry (NCS) averaging, starting from a cryo-electron microscopy reconstructed model (Kato et al., 2008). Despite the strength of averaging strongly supporting the similarity of all of the MVP subunits, the 39 monomers contained in the crystal asymmetric unit were independently built and explicitly used during refinement. The relaxation of the 39-fold rotational symmetry resulted in some movements and conformational differences between MVP monomers and greatly increased the number of degrees of freedom (Tanaka et al., 2009). Almost in parallel, the $2.1 \AA$ resolution structure of the seven $\mathrm{N}$-terminal repeat domains of MVP (R1-7), which form the vault waist, was reported together with an independent determination of the vault structure at $8 \AA$ resolution, which had again been phased by density modification with averaging (Querol-Audí et al., 2009). The structures provided some hints on the vault opening mechanism, showing that a combination of electrostatic and hydrophobic interactions, mediated by R1 repeats, govern the association of the two half-vault moieties. This observation led to the proposal of a reversible mechanism of dissociation of the vault particle induced at acidic $\mathrm{pH}$.

In this work, we have generated a new refined model of the vault particle by incorporating the high-resolution structure of the MVP N-terminal domain (R1-7; Querol-Audí et al., 2009) into the $3.5 \AA$ resolution model (Tanaka et al., 2009). A new refinement of the vault structure was performed on a single MVP subunit, strictly maintaining the 39-fold noncrystallographic symmetry. An extension of the deformable elastic network (DEN) approach was employed, as included in the low-resolution refinement package of $C N S$ v.1.3 (Schröder et al., 2010). The structural analysis of the refined vaults,

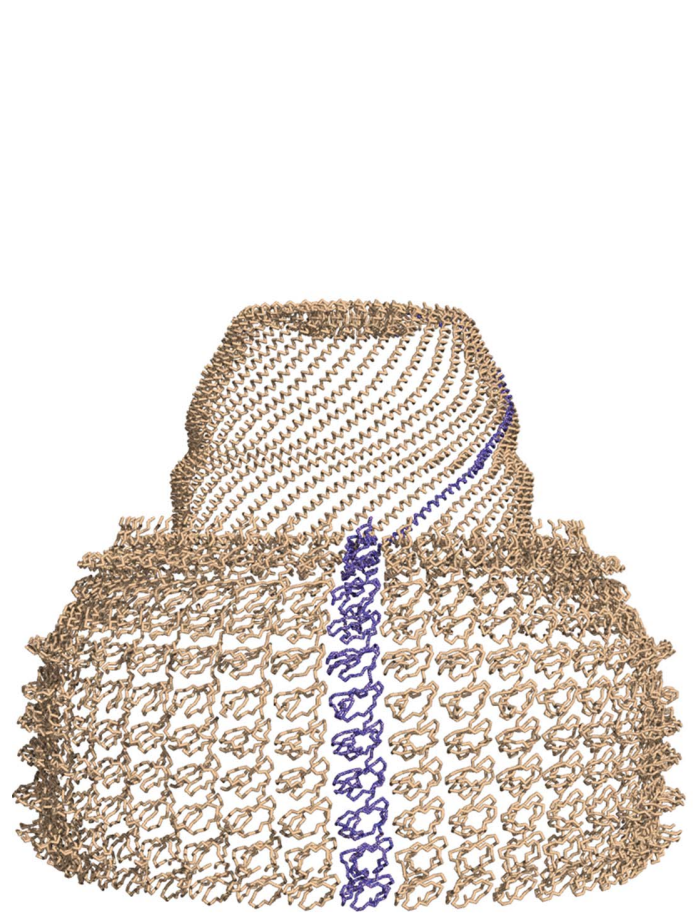

(a)

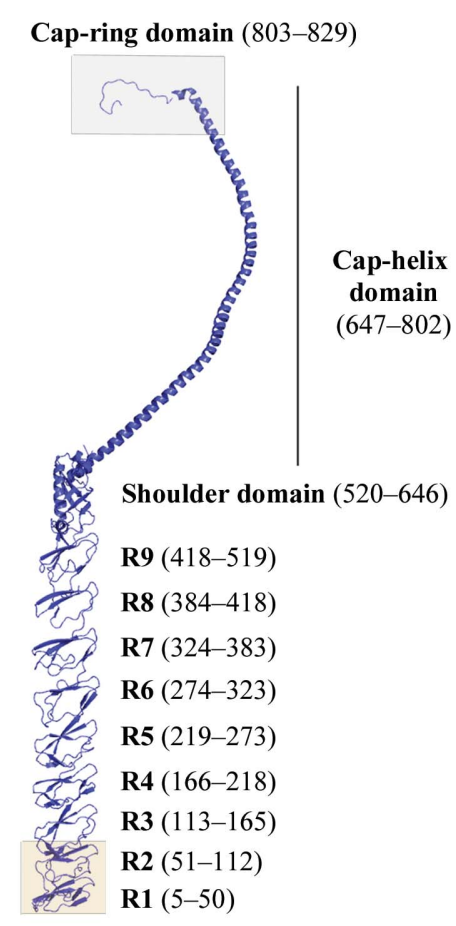

(b)

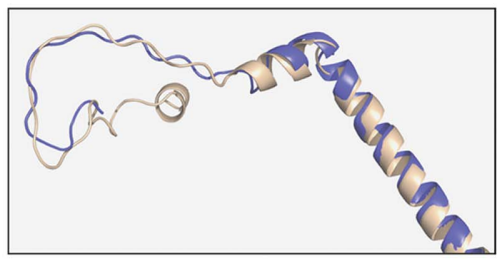

(c)

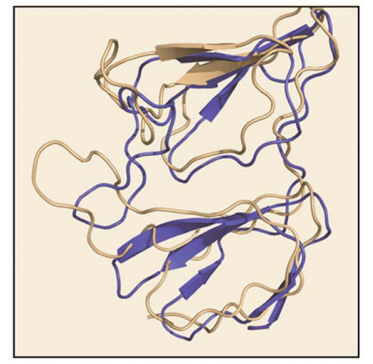

(d)

Figure 1

Overall view of the vault structure refined using DEN protocols. (a) Half-vault structure (in beige) with one of the 39 MVP copies forming the particle shown in blue. (b) Ribbon representation of one MVP monomer showing the overall fold. The 12 structural domains are explicitly labelled (sequence numbering is shown for the starting and final residues of each domain). (c) Close-up of the cap-ring showing the superimposition of the new refined MVP structure with the same part of subunit $A$ from the structure with PDB code 2zuo (beige). (d) Close-up of the R1-2 repeats also showing the superimposition of the two refined models of this MVP region. 
facilitated now by having only one independent MVP subunit, suggests that MVP can experience important movements at its $\mathrm{N}$-terminus during the closing of the two vault halves. It also provides significant insights into the cap-ring architecture, including a potential role in the binding of other vault components and binding partners.

\section{Materials and methods}

\subsection{Vault refinement using 39-fold symmetry and DEN protocols}

The MVP hybrid structure (residues 4-380 of PDB entry 3 gnf plus residues $381-814$ of PDB entry $2 \mathrm{zv} 4$ ) was refined against the experimental structure factors from the work of Tanaka et al. (2009) (PDB entry 2zv4). Refinement was performed using an extension of the deformable elastic network (DEN) approach included in the $C N S$ v.1.3 package (Schröder et al., 2010). During refinement, 39-fold noncrystallographic symmetry (NCS) was always strictly maintained with refinement parameters restricted to a single MVP subunit and using the skew-matrix formalism also available in $C N S$.

\subsection{The skew-matrix formalism}

NCS position $A_{1}^{\prime}$ is related to input coordinates $A_{1}$ according to

$$
A_{1}^{\prime}=\mathbf{R} A_{1}+\mathbf{T}
$$

where $\mathbf{R}$ is a general rotation matrix, $\mathbf{T}$ a translation vector and the subscript 1 indicates the crystal reference frame.

The skew formalism firstly allows the oligomeric particle (in our case the half vault) to be built in a standard orientation where the NCS relations have simple forms, and secondly positions the particle as a whole (the half vault) in its location within the unit cell by a skew transformation that, in general, corresponds to a skew rotation (matrix $\mathbf{P}$ ) and a skew translation. In our case, with the vault half related by the crystallographic twofold at the $y$ axis of a monoclinic space group, the rotational axis of the particle passes through the origin; consequently, translations, both in the standard and in the crystal systems, are null and will be omitted.

Therefore,

$$
A_{1}=\mathbf{P} A_{2}
$$

where the subscript 2 indicates coordinates in the standard orientation,

$$
A_{2}^{\prime}=\mathbf{U} A_{2},
$$

where $\mathbf{U}$ is an NCS rotational matrix in the standard orientation,

$$
\begin{gathered}
A_{2}^{\prime}=\mathbf{U} \mathbf{P}^{-1} A_{1}, \\
A_{1}^{\prime}=\mathbf{P} A_{2}^{\prime}=\mathbf{P} \mathbf{U} \mathbf{P}^{-1} A_{1}=\mathbf{R} A_{1},
\end{gathered}
$$

where $\mathbf{R}=\mathbf{P} \mathbf{U} \mathbf{P}^{-1}$.

\subsection{The DEN protocol}

A low-resolution refinement protocol based on the deformable elastic network (DEN) approach (Schröder et al., 2010) included in the CNS v.1.3 package was used. In this protocol the model-refinement parameters are not the atomic coordinates but the torsion angles, which reduces the number of degrees of freedom by a factor generally larger than five. The 39-fold noncrystallographic symmetry was strictly maintained during refinement with constraints applied to a single MVP monomer, which was generated as a hybrid model containing the R1-7 structure at the $\mathrm{N}$-terminus (residues Glu4-Val380) and coordinates corresponding to monomer $A$ of the deposited MVP model at the C-terminus (PDB entry 2zv4; residues Pro381-Gly814). The structural information available for the R1-7 fragment was also used to increase the amount of stereochemical data. This approach provided the stability that the initial refinement attempts had been lacking, with a reduction of about $\sim 200$ times $(\sim 5 \times 39)$ in the number of degrees of freedom with respect to the published $3.5 \AA$ vault structure and a significant increment in the number of stereochemical restraints. The resulting low-resolution refinement maps were often combined with averaging and solvent-flattening processes.

\subsection{Averaging and solvent flattening}

Density averaging and solvent flattening were applied with the program DM (Cowtan \& Main, 1998) using the deposited experimental data (PDB entry 2zv4) and different MVP models for initial phasing.

The 39 original rotation matrices (in the standard orientation) multiplied by the skew matrix provided the 39 transformations, all with null translations, required by $D M$. The resulting maps allowed us to detect relatively important changes in the domains of the R1-7 region between the structures of the fragment and of the intact vault. Cycles of $C N S$ and $D M$ were combined with manual model building with Coot. To remove model bias, many different densitymodification processes were performed with starting phases obtained from truncated models (for example, with only main chain, without a specific repeat etc.).

\subsection{Map sharpening}

The contribution of the high-resolution diffraction data to the electron density was enhanced by map sharpening, which progressively up-weights the structure factors at increasing resolutions by applying a negative $B_{\text {sharp }}$ value to the experimental amplitudes $\left(F_{\text {obs }}\right)$ in a resolution-dependent weighting scheme (Brunger et al., 2009),

$$
F_{\text {sharpened }}=\exp \left[-B_{\text {sharp }} \sin ^{2}(\theta) / \lambda^{2}\right] F_{\text {obs }},
$$

where $\theta$ is the Bragg angle and $\lambda$ is the wavelength.

Despite the fact that sharpening generally increases the noise in the resulting electron-density maps, it appears this can in turn be significantly reduced by density modification, particularly by density averaging. 
research papers

\section{Results and discussion}

\subsection{Generation of a new MVP vault particle model}

To incorporate the high-resolution information available from the R1-7 crystal structures (Querol-Audí et al., 2009) into the structure of the vault, a preliminary hybrid model of an MVP subunit was generated from the coordinates corresponding to monomer $A$ of the $3.5 \AA$ resolution vault structure (Tanaka et al., 2009; PDB entry 2zv4), in which the R1-7 repeats were replaced by the corresponding ones from the R1-7 crystal structures (Querol-Audí et al., 2009). The three crystal structures available for R1-7 (at 3.0, 2.5 and $2.1 \AA$ resolution; PDB entries 3gng, 3gf5 and 3gnf, respectively; Querol-Audí et al., 2009) presented almost identical conformations except for a few residues at the $\mathrm{N}$-terminus. Therefore, the R1-7 model with the highest resolution (2.1 $)$ ), which included residues Glu4-Val380, was used to replace the R1-7 repeats. Each of the seven repeats was independently superimposed as the largest differences were owing to changes in their relative orientations, in particular for repeats R1, R2 and R7 (Querol-Audí et al., 2009). Superimpositions were performed with $L S Q K A B$ from the CCP4 package (Winn et $a l ., 2011$ ), except for repeats $\mathrm{R} 1$ and $\mathrm{R} 2$, which were superimposed using the molecular-replacement program MOLREP (Vagin \& Teplyakov, 2010) owing to large discrepancies in the tracing and the lengths of the loops and in the assignment of side chains when compared with those in the context of the vault (Querol-Audí et al., 2009).

The resulting new model of the MVP subunit (including residues Glu4-Gly814), with all of the atomic temperature factors initially set to an arbitrary value $\left(30 \AA^{2}\right.$ ), was subjected to refinement using the deposited $3.5 \AA$ resolution experimental data (Tanaka et al., 2009; PDB entry 2zv4), assuming strict 39-fold noncrystallographic symmetry (NCS) for the half-vault particle contained in the crystal asymmetric unit. The complete vault particle was then obtained by the application of a crystallographic twofold axis at the origin (monoclinic space group $C 2$ ) that is coincident with one of the particle twofold axes. In the first refinement step the whole MVP subunit was treated as a single rigid body to overcome any possible bias related to having arbitrarily chosen monomer $A$ from the 39 possible alternatives in the $3.5 \AA$ resolution deposited vault structure (PDB entries 2zv4, 2zuo and 2zv5). After convergence, different combinations of the MVP domains were allowed to refine as independent rigid bodies. The small distortions of connectivity introduced into the model by the splitting into domains during the rigid-body refinements were smoothed and regularized using Coot (Emsley et al., 2010) and the regularize option of CNS (Brünger et al., 1998). During these refinements the 39-fold noncrystallographic rotational symmetry was always strictly maintained and fixed in the orientation that had been derived previously during the vault structure determination: with the 39-fold rotational axis passing throughout the unit-cell origin perpendicular to the crystal twofold $b$ axis ( $y$ axis) and making an $\varphi$ angle of $70.17^{\circ}$ with respect to the unit-cell $a$ axis ( $x$ axis) (Tanaka et al., 2009). Once a model of the new MVP vault shell
Table 1

Refinement of the orientation angle $\varphi$.

\begin{tabular}{llllll}
\hline Angle $\varphi\left(^{\circ}\right)$ & 70.14 & 70.15 & 70.16 & 70.17 & 70.18 \\
\hline$R$ factor & 0.45721 & 0.45699 & 0.45693 & 0.45707 & 0.45778 \\
\hline
\end{tabular}

Table 2

Refinement statistics of the vault model using strict 39-fold NCS averaging: comparison with unrestricted refinement.

\begin{tabular}{|c|c|c|}
\hline & $\begin{array}{l}\text { One MVP monomer } \\
\text { and } 39 \text {-fold NCS } \\
\text { symmetry }\end{array}$ & $\begin{array}{l}39 \text { MVP monomers } \\
\text { (Tanaka et al., 2009) }\end{array}$ \\
\hline Resolution ( $\mathrm{A})$ & $204-3.5(3.63-3.50)$ & $204-3.5$ \\
\hline Reflections used & $1441463 \dagger$ & 1436395 \\
\hline No. of protein residues & 796 & 31668 \\
\hline$R_{\text {work }}(\%)$ & $0.352(0.488)$ & 0.311 \\
\hline$R_{\text {free }}(\%)$ & $0.355(0.474)$ & 0.330 \\
\hline \multicolumn{3}{|l|}{ R.m.s.d. from ideal } \\
\hline Bond lengths $(\AA)$ & 0.003 & 0.010 \\
\hline Bond angles $\left({ }^{\circ}\right)$ & 0.84 & 1.48 \\
\hline \multicolumn{3}{|c|}{ Ramachandran plot statistics (\%) } \\
\hline Most favoured region & 91.4 & 80.0 \\
\hline Additionally allowed region & 8.4 & 18.3 \\
\hline Generously allowed region & 0 & 1.4 \\
\hline Disallowed region & $0.2 \ddagger$ & 0.3 \\
\hline \multicolumn{3}{|l|}{ Averaged $B$ factors $\left(\AA^{2}\right)$} \\
\hline Main chain & 143.0 & 110.2 \\
\hline Side chain & 145.5 & 133.1 \\
\hline Overall & 144.2 & 121.4 \\
\hline
\end{tabular}

$\dagger$ Reflections with $[F / \sigma(F)]>2.2$ were used in the refinement for comparative consistency with Tanaka et al. (2009). $\ddagger$ Corresponding to two proline residues located in two exposed loops.

was available the direction of the rotational axis was crosschecked by a systematic search, obtaining slightly better agreement values for a $\varphi$ value of $70.16^{\circ}$ (Table 1 ). All of these rigid-body refinements were facilitated by the skew-matrix formalism available in $C N S$ (see $\$ 2$ ). This formalism allows the vault particle to first be built in a standard orientation (chosen here as that with the 39 -fold rotational symmetry axis along the unit-cell axis $a$, the $x$ axis of the reference frame), where the NCS relations have simple forms [here, pure rotations around the $x$ axis by angles $n \times(360 / 39)^{\circ}$, with $n=1,2, \ldots$, 39]. In a second step the vault is positioned in its true location within the unit cell by a transformation including, in general, a rotation (here around the $y$ axis by angle $\varphi$ ) and a translation (which is null here).

At the resolution available, maintaining strict NCS was considered to be essential during refinement for both conceptual and practical reasons. From the analysis of the packing it was difficult to rationalize a systematic distortion of the 39-fold NCS that could be well maintained in the monoclinic crystal, even more so as it seems to be a subtle distortion at most and capable of retaining some of the twofold particle symmetries as required by the coincidence with the crystal twofold symmetry. Given the power of averaging and the quality of the averaged maps, the expected differences between MVP subunits should be, at $3.5 \AA$ resolution, at most very small. At this (low) resolution the number of degrees of freedom approaches the number of reflections when explicitly using the coordinates and temperature factors of the $39 \mathrm{MVP}$ 
subunits contained in the crystal asymmetric unit. The analysis, and even the handling, of a model containing 39 MVP subunits is cumbersome, starting with the need for three different PDB entries to store the coordinates. The quality of the electron density obtained in this work upon application of strict NCS provides additional validation of the procedure used.

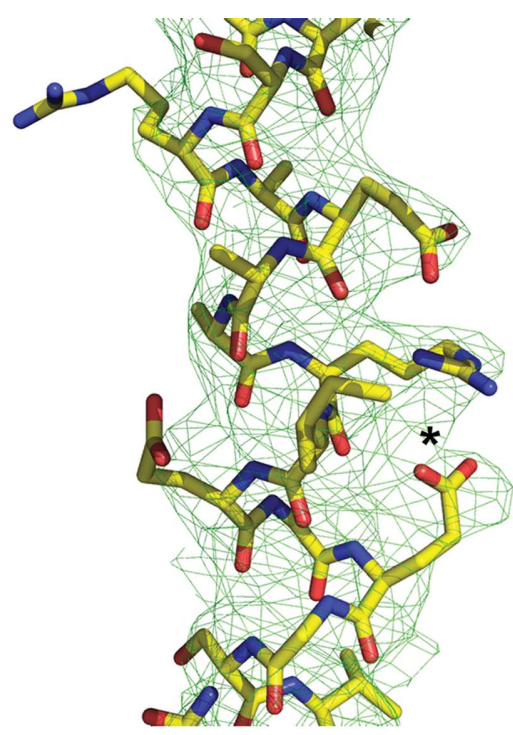

(a)
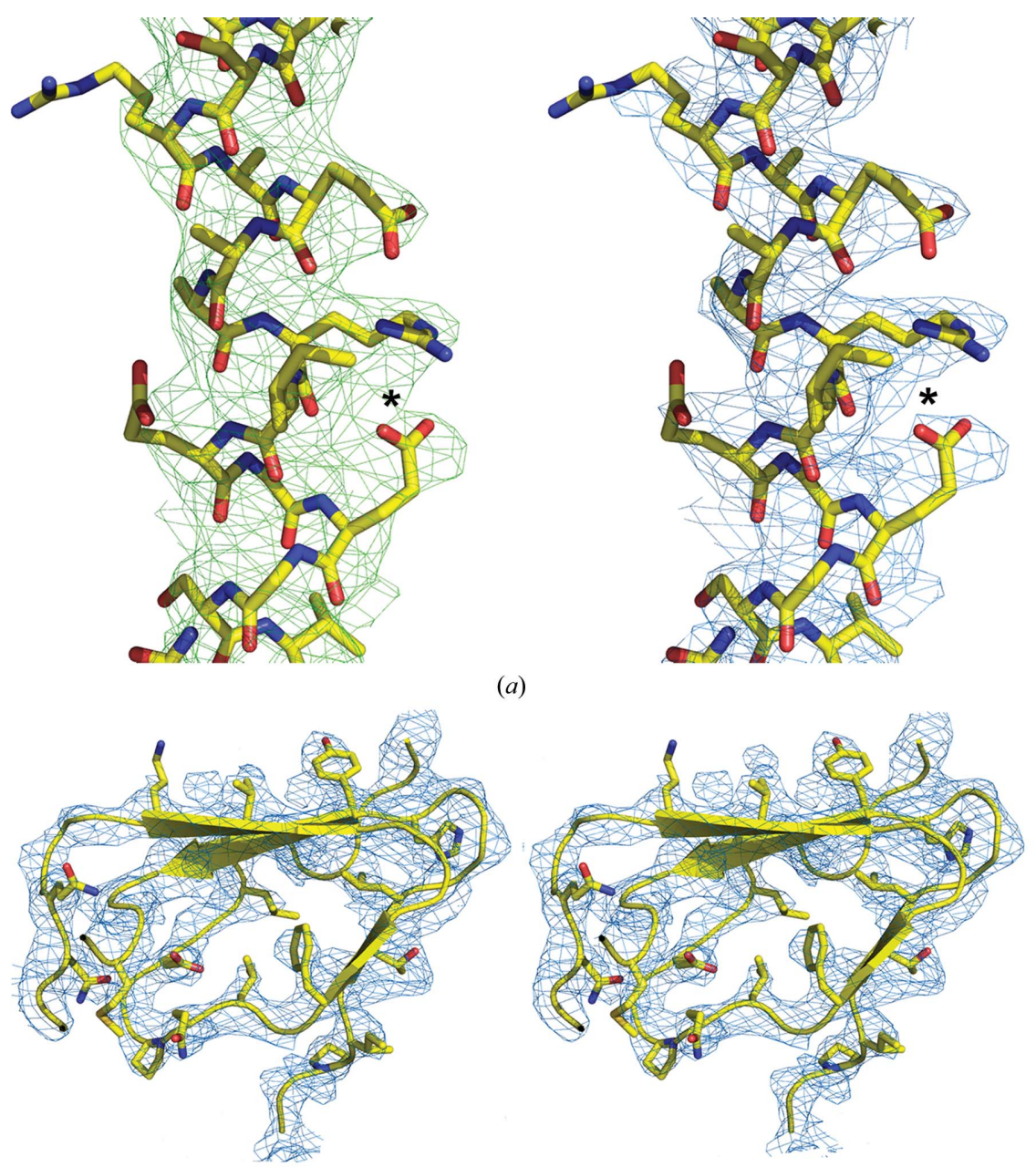

(b)
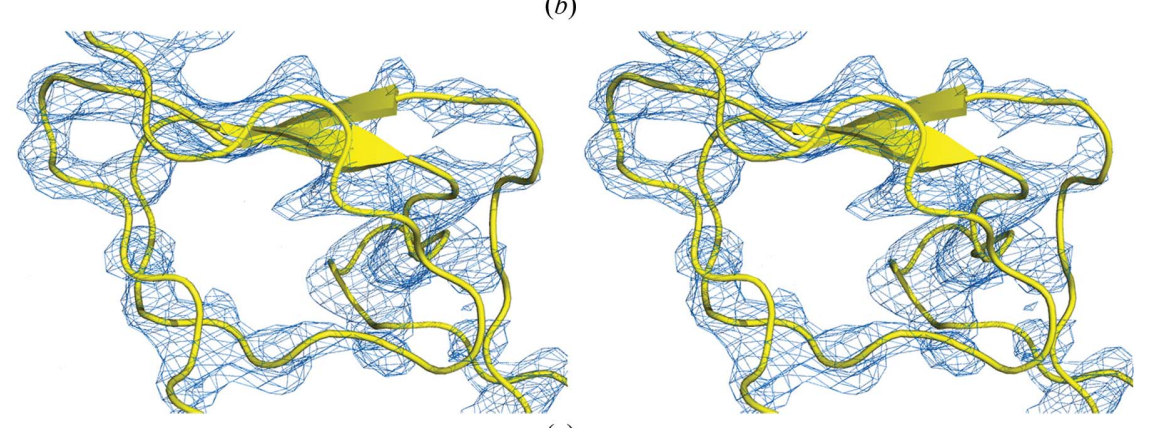

Figure 2

(c)

Map sharpening enhances the high-resolution details of the maps. (a) Electron-density maps (contoured at 2.5 $\sigma$ ) before (green) and after (blue) map sharpening corresponding to the caphelix domain of the MVP (amino acids 728-741). Polypeptides are represented as sticks. $(b)$ Stereoscopic view of the electron-density map of repeat R6 (contoured at 2.5\%) after refinement using DEN protocols. The structure of the repeat is depicted as ribbons, with some of the side chains drawn as sticks. (c) Stereoscopic view of the electron-density map of repeat R2 (depicted at the $1.3 \sigma$ level). Repeat R2 is shown as ribbons. Density at the vault mid-section is less well defined than for the rest of the structure.
Refinement was continued with the refine protocols from $C N S$ v.3.1 using the deformable elastic network approach and always maintaining strict NCS. The final refinement cycles converged to an $R_{\text {free }}$ of $\sim 35 \%$ for 1441463 reflections (Table 2). The fact that the new model gives a slightly higher $R_{\text {free }}$ than the previous model $(\sim 33 \%$; Table 2$)$ is not surprising considering the enormous reduction in the number of degrees of freedom in this new refinement (from 960942 parameters in the all-atom refinement to 2955 parameters when using torsional angles and strict NCS in the new refinement protocol; see $\$ 2$ ). The quality of the final new model and the corresponding refinement statistics are summarized and compared with the available vault model in Table 2. The electron-density maps were further modified by enhancing the contribution of the diffraction data at high resolution by applying map sharpening with

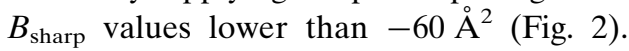
Side chains and parts of MVP were removed at different stages to monitor and minimize model bias during sharpening (data not shown).

\subsection{Structural analysis}

The resulting electron-density maps present clear high-resolution secondarystructure features (Figs. $2 a$ and $2 b$ ). However, density for R1, and to some extent $\mathrm{R} 2$, is less well defined than for the remaining repeats (Fig. $2 c$ ), which could be related to the difficulties in tracing $\mathrm{R} 1-2$ in the published $3.5 \AA$ resolution vault structure and raises the possibility of some disorder in the vault waist region, close to the vault midsection, that could be blurring the electron-density maps. Also, density for the C-terminal part of the protein, after Pro815, was difficult to interpret. The description of the MVP subunit as constituted of ten globular domains (nine repeat domains and a shoulder domain) and two C-terminal nonglobular regions (a long caphelix and the cap-ring) remains fully valid in the present refinement. In particular, from R3 to the cap-helix the structure of the new MVP model remains nearly identical to the published structure (Tanaka et al., 2009), although there are several sequence shifts by one or a few positions in the R3-7 region. Interestingly, the $\beta 2-\beta 3$ loop of $\mathrm{R} 7$ is well defined in the structure of the intact vault but not in the R1-7 crystal structures. Reevaluation of the vault midsection, owing to the retracing of $\mathrm{R} 1$ and $\mathrm{R} 2$, agrees with the 
analysis that led us to propose a mechanism of dissociation of the vault particle induced by a $\mathrm{pH}$ change (Querol-Audí et al., 2009).

In the cap-ring the electron density first points towards the rotational axis of the particle, while at the smallest radius $(\sim 25 \AA$ ) it runs parallel to the axis (Fig. 1). Packing at this small radius would allow at most 36 copies of parallel $\beta$-strands with a closest distance between adjacent strands of $\sim 4.5 \AA$ (Figs. $3 a$ and $3 b$ ). All of the prediction algorithms used [T-Coffee (Notredame et al., 2000), JPred3 (Cole et al., 2008) and ESPript (Gouet et al., 1999)] indicate the presence of a conserved short $\beta$-strand corresponding to the MVP sequence Leu822-Leu825 in the cap-region. Similar sequences can be found in at least 13 of the $\beta$-strands forming the 16 -stranded parallel $\beta$-sheet of the horseshoe-shaped (leucine-rich repeat) ribonuclease inhibitor (Fig. 3c; PDB entry 1dfj; Kobe \& Deisenhofer, 1993). Therefore, a ring-like $\beta$-sheet with 36

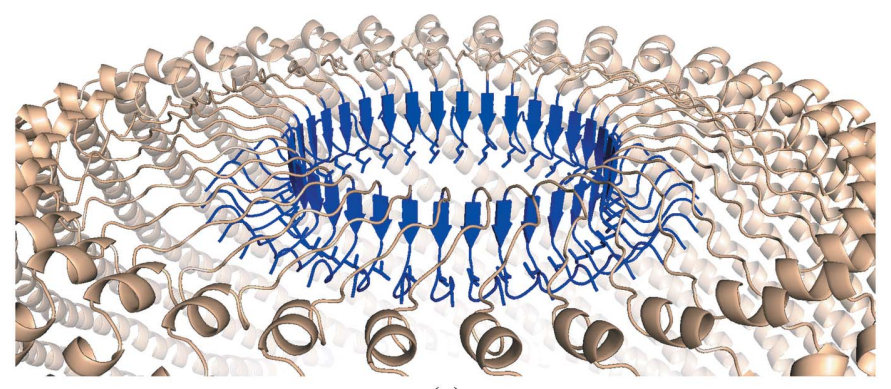

(a)

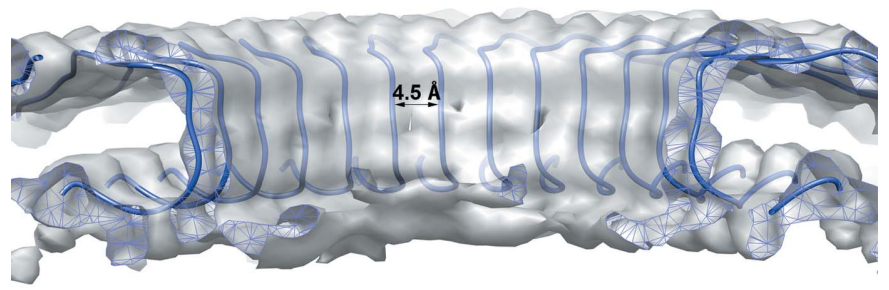

(b)

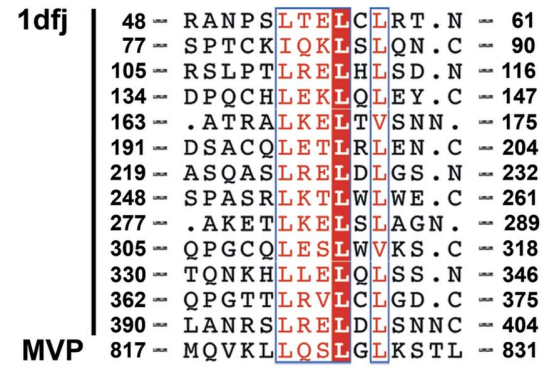

(c)

Figure 3

(a) Representation of a possible arrangement of the cap-region with the 36 -stranded $\beta$-sheet inner ring (radius of $\sim 25 \AA$ ) shown in blue and the 39 subunits in the outer part shown in beige. Therefore, three MVP subunits appear to adopt alternative conformations avoiding the inner ring (see text). (b) View of the continuous electron density that forms the vault cap-ring. The tracing of only 13 chains is shown for clarity, with the distance within strands explicitly labelled. (c) Sequence alignment of 13 $\beta$-strands (from the 16 forming the parallel $\beta$-sheet of the ribonuclease inhibitor structure; PDB entry $1 \mathrm{dfj}$ ) with the corresponding region of the C-terminus of MVP. Residue numbers are indicated for each sequence and the predicted $\beta$-strand for MVP is drawn at the bottom. parallel strands was built at the vault inner cap-ring with the guidance of the $\beta$-sheet found in the ribonuclease inhibitor structure (Fig. 3a). The three (or more) MVP subunits excluded from the $\beta$-sheet ring have to adopt alternative conformations outside this inner cap-ring region. The possibility of a compact structure not following 39 -fold symmetry (with 36, or maybe fewer, subunits) is compatible with the presence in the cap of continuous electron density where separation between subunits is not recognizable. The numerous contacts between adjacent MVP subunits within the half-vault could still guarantee the stability of the particle. Moreover, MVP variants with C-terminal extensions produce vault-like structures with the extensions pointing out of the vault structure (Kickhoefer et al., 2009). In conclusion, packing and electron-density maps strongly suggest that on average one in every 13 MVP subunits presents an alternative conformation outside the cap-ring.

\subsection{Structural comparisons}

Despite the overall similarity of the N-terminal repeats in the new refined MVP model to those in the R1-7 crystal structures, there are significant changes in the relative orientations of the repeats with respect to each other, in particular for R1-2, for R6 and for R7 (Fig. 4). On the other hand, the orientations of the repeats remain essentially unchanged in the three available R1-7 crystal structures, indicating that these orientations are not determined by crystal-packing interactions. Therefore, the different orientations of the repeats in the MVP structure and in the R1-7 fragments should be related to specific interactions in the vault that are absent, or different, in the R1-7 fragments. The altered environment at the $\mathrm{C}$-end of the R1-7 fragment with respect to an intact MVP subunit could explain, at least in part, the large difference observed for the orientation of the R7 repeat, which in turn also affects R6 and, to a lesser extent, R5 and so on. In contrast, the different orientations of the $\mathrm{N}$-end repeats have to be a consequence of differences in the interactions between neighbouring MVP subunits in the intact vault and in the R1-7 crystals. In particular, interactions between the R1 repeats from the two vault halves are absent in the R1-7 crystals and could explain the large differences observed in the orientations of the $\mathrm{R} 1$ and $\mathrm{R} 2$ repeats. What could the biological implications of the N-terminus of MVP presenting a twisted conformation in a vault environment with respect to an isolated subunit be? The latest reviews of vault biology have come to accept that this particle must have important functions as an intracellular transport vehicle (Berger et al., 2009; Steiner et al., 2006). Several proteins of considerable size (PTEN, Erk etc.) have been identified as being bound and some of them also as being transported by MVP (reviewed in Berger et al., 2009). If these cargoes have to be protected in the vault interior, an efficient mechanism of vault opening will be required. Morever, vPARP has already been localized to bind to the interior of the vault structure (Mikyas et al., 2004) and can be incorporated into pre-formed vault-like structures (Poderycki et al., 2006), indicating that this particle is not as 
hermetic as it might appear. The opening of the vault particle might be triggered by molecular breathing (although this has not yet been reported) or by an acidic $\mathrm{pH}$ inducing the
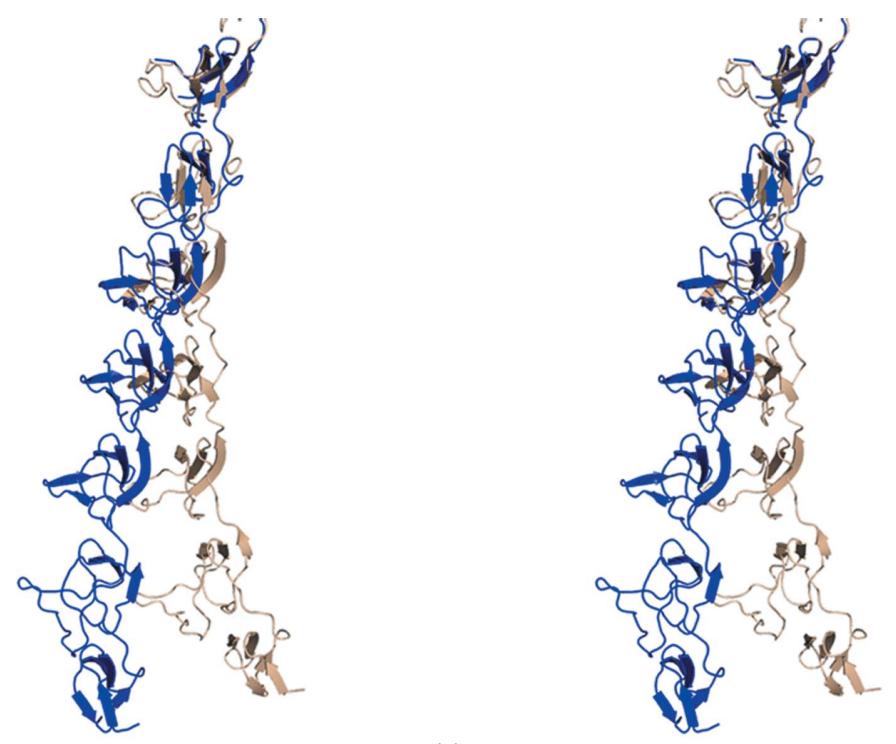

(a)
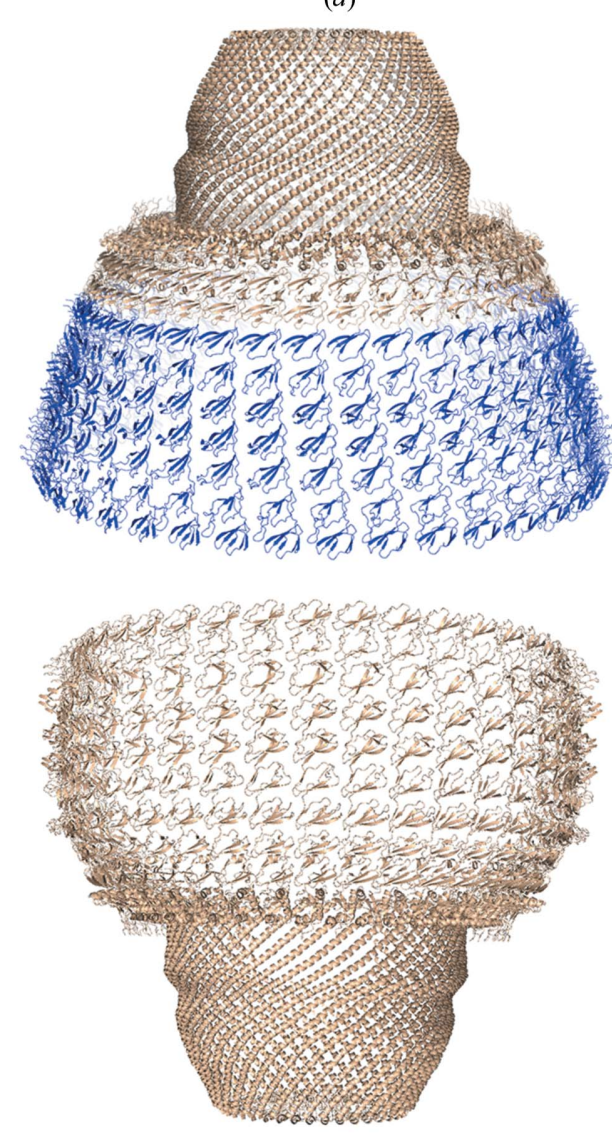

(b)

\section{Figure 4}

The R1-7 region presents a more closed conformation in the vault particle when compared with the R1-7 soluble domain. (a) Stereoview of the superposition of repeat R7 in the structure of the R1-7 soluble domain ring (blue) on the corresponding region within the vault (beige). (b) A hybrid half-vault model generated by the superposition displayed in $(a)$ in the context of the vault particle is shown at the top and the usual closed half vault is shown below. protonation of key acidic residues that could then initiate the separation of the vault halves by charge repulsion (QuerolAudí et al., 2009). In any case, the breakage of the midsection contacts is now expected to trigger movement of the R1-2 repeats towards the orientation observed in the R1-7 fragments, thus further facilitating the cooperative separation of the two halves (Fig. 4b) as has been described to occur for the vault particle (Yang et al., 2010).

The C-terminal region of the cap-ring, which presents a twolayered density, remains difficult to interpret. Therefore, after Pro815 the tracing with just $\mathrm{C}^{\alpha}$ atoms indicates that the likely chain path was retained (Tanaka et al., 2009). However, the extended density joining the two layers corresponds very well to a four-residue $\beta$-strand that would form an intersubunit parallel $\beta$-sheet, likely corresponding to residues Leu822Leu825 (Fig. 3c). This would require small rearrangements in the trace from Pro 815 to Leu822 and would also imply that the final $\mathrm{C}^{\alpha}$ position corresponds to residue 829 (Fig. $1 b$ ). This intersubunit parallel $\beta$-sheet, which is likely to be formed by only 36 strands, defines the narrowest radius $(\sim 25 \AA)$ of the MVP shell with respect to the central vault axis. It is interesting to remark that in the ribonuclease inhibitor there is a rim of exposed hydrophobic residues on the internal face of the $\beta$-sheet ring where ribonuclease binds (Kobe \& Deisenhofer, 1993, 1995). In the $\beta$-sheet ring of vaults a similar exposed hydrophobic rim could be formed by Leu 825 and be directed towards the unexplained central density that might correspond to a disordered TEP1 (Tanaka et al., 2009).

\section{Conclusion}

The precise analysis of the vault structure was complicated by the presence of 39 independent MVP subunits with slightly different conformations and interactions, with a total number of 31668 protein residues corresponding to 810 residues in each of the 39 MVP subunits. The structural analysis of vaults is now simplified by a model defined by one independent MVP subunit and corresponding strict NCS-related molecules. This analysis reveals that important movements occur at the $\mathrm{N}$-terminus of MVP during the closing of the two vault halves. The analysis also predicts the breakage of the 39-fold symmetry in the cap-region (residues 815-849). A plausible conformation similar to that of the ribonuclease inhibitor indicates that the cap-ring intersubunit parallel $\beta$-sheet might act as a switch to precisely define the number of MVP subunits in a vault and as a potential binding site for the other vault components and binding partners.

This work was supported in part by a Grant-in-Aid for Scientific Research (21227003; to TT), a Grant-in-Aid for Young Scientists (A) (24687020; to HT) and a Grant-in-Aid for Scientific Research on Innovative Areas (23121517; to HT) from the Ministry of Education, Culture, Sports, Sciences and Technology of Japan and a PRESTO program grant from the Japan Science and Technology Agency (to HT). This work was also supported by grants from the Ministerio de Economia y Competitividad of Spain to NV (BIO2011-24333) and to IF 
(BFU2012-36827). Many thanks are given to the PDB for valuable help during the validation process.

\section{References}

Berger, W., Steiner, E., Grusch, M., Elbling, L. \& Micksche, M. (2009). Cell. Mol. Life Sci. 66, 43-61.

Brünger, A. T., Adams, P. D., Clore, G. M., DeLano, W. L., Gros, P., Grosse-Kunstleve, R. W., Jiang, J.-S., Kuszewski, J., Nilges, M., Pannu, N. S., Read, R. J., Rice, L. M., Simonson, T. \& Warren, G. L. (1998). Acta Cryst. D54, 905-921.

Brunger, A. T., DeLaBarre, B., Davies, J. M. \& Weis, W. I. (2009). Acta Cryst. D65, 128-133.

Cole, C., Barber, J. D. \& Barton, G. J. (2008). Nucleic Acids Res. 36, W197-W201.

Cowtan, K. \& Main, P. (1998). Acta Cryst. D54, 487-493.

Emsley, P., Lohkamp, B., Scott, W. G. \& Cowtan, K. (2010). Acta Cryst. D66, 486-501.

Esfandiary, R., Kickhoefer, V. A., Rome, L. H., Joshi, S. B. \& Middaugh, C. R. (2009). J. Pharm. Sci. 98, 1376-1386.

Goldsmith, L. E., Yu, M., Rome, L. H. \& Monbouquette, H. G. (2007). Biochemistry, 46, 2865-2875.

Gouet, P., Courcelle, E., Stuart, D. I. \& Métoz, F. (1999). Bioinformatics, 15, 305-308.

Hamill, D. R. \& Suprenant, K. A. (1997). Dev. Biol. 190, 117-128.

Herlevsen, M., Oxford, G., Owens, C. R., Conaway, M. \& Theodorescu, D. (2007). Mol. Cancer Ther. 6, 1804-1813.

Herrmann, C., Zimmermann, H. \& Volknandt, W. (1997). Gene, 188, 85-90.

Kato, K., Tanaka, H., Sumizawa, T., Yoshimura, M., Yamashita, E., Iwasaki, K. \& Tsukihara, T. (2008). Acta Cryst. D64, 525-531.

Kedersha, N. L., Miquel, M. C., Bittner, D. \& Rome, L. H. (1990). J. Cell Biol. 110, 895-901.

Kedersha, N. L. \& Rome, L. H. (1986). J. Cell Biol. 103, 699-709.

Kickhoefer, V. A., Han, M., Raval-Fernandes, S., Poderycki, M. J., Moniz, R. J., Vaccari, D., Silvestry, M., Stewart, P. L., Kelly, K. A. \& Rome, L. H. (2009). ACS Nano, 3, 27-36.

Kickhoefer, V. A., Searles, R. P., Kedersha, N. L., Garber, M. E., Johnson, D. L. \& Rome, L. H. (1993). J. Biol. Chem. 268, 78687873.
Kickhoefer, V. A., Siva, A. C., Kedersha, N. L., Inman, E. M., Ruland, C., Streuli, M. \& Rome, L. H. (1999). J. Cell Biol. 146, 917-928.

Kickhoefer, V. A., Stephen, A. G., Harrington, L., Robinson, M. O. \& Rome, L. H. (1999). J. Biol. Chem. 274, 32712-32717.

Kobe, B. \& Deisenhofer, J. (1993). Nature (London), 366, 751-756.

Kobe, B. \& Deisenhofer, J. (1995). Nature (London), 374, 183-186.

Kowalski, M. P., Dubouix-Bourandy, A., Bajmoczi, M., Golan, D. E., Zaidi, T., Coutinho-Sledge, Y. S., Gygi, M. P., Gygi, S. P., Wiemer, E. A. C. \& Pier, G. B. (2007). Science, 317, 130-132.

Mikyas, Y., Makabi, M., Raval-Fernandes, S., Harrington, L., Kickhoefer, V. A., Rome, L. H. \& Stewart, P. L. (2004). J. Mol. Biol. 344, 91-105.

Notredame, C., Higgins, D. G. \& Heringa, J. (2000). J. Mol. Biol. 302, 205-217.

Poderycki, M. J., Kickhoefer, V. A., Kaddis, C. S., Raval-Fernandes, S., Johansson, E., Zink, J. I., Loo, J. A. \& Rome, L. H. (2006). Biochemistry, 45, 12184-12193.

Querol-Audí, J., Casañas, A., Usón, I., Luque, D., Castón, J. R., Fita, I. \& Verdaguer, N. (2009). EMBO J. 28, 3450-3457.

Scheffer, G. L., Wijngaard, P. L., Flens, M. J., Izquierdo, M. A., Slovak, M. L., Pinedo, H. M., Meijer, C. J., Clevers, H. C. \& Scheper, R. J. (1995). Nature Med. 1, 578-582.

Schröder, G. F., Levitt, M. \& Brunger, A. T. (2010). Nature (London), 464, 1218-1222.

Stadler, P. F., Chen, J. J.-L., Hackermüller, J., Hoffmann, S., Horn, F., Khaitovich, P., Kretzschmar, A. K., Mosig, A., Prohaska, S. J., Qi, X., Schutt, K. \& Ullmann, K. (2009). Mol. Biol. Evol. 26, 19751991.

Steiner, E., Holzmann, K., Elbling, L., Micksche, M. \& Berger, W. (2006). Curr. Drug Targets, 7, 923-934.

Tanaka, H., Kato, K., Yamashita, E., Sumizawa, T., Zhou, Y., Yao, M., Iwasaki, K., Yoshimura, M. \& Tsukihara, T. (2009). Science, 323, 384-388.

Tanaka, H. \& Tsukihara, T. (2012). Proc. Jpn Acad. Ser. B Phys. Biol. Sci. 88, 416-433.

Vagin, A. \& Teplyakov, A. (2010). Acta Cryst. D66, 22-25.

Winn, M. D. et al. (2011). Acta Cryst. D67, 235-242.

Yang, J., Kickhoefer, V. A., Ng, B. C., Gopal, A., Bentolila, L. A., John, S., Tolbert, S. H. \& Rome, L. H. (2010). ACS Nano, 4, 72297240 . 